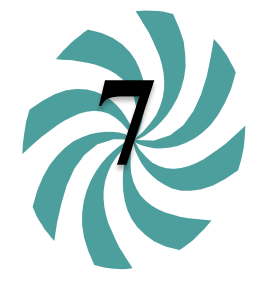

Tecnociencia, Vol. 22, N²: 123-149

julio-diciembre 2020

\title{
FIRST ANNOTATED CHECKLIST OF BIRDS OF JICARÓN AND JICARITA: THE SOUTHERNMOST ISLANDS OF THE REPUBLIC OF PANAMA
}

\author{
${ }^{1,2}$ Pedro Luis Castillo-Caballero,,${ }^{1,3,4}$ Claudio M. Monteza-Moreno, ${ }^{5}$ Oscar \\ Johnson, \& ${ }^{1}$ George R. Angehr \\ ${ }^{1}$ Smithsonian Tropical Research Institute, Ancon, Panama, Republic of Panama \\ ${ }^{2}$ Escuela de Biología, Universidad de Panama, Panama, Republic of Panama \\ ${ }^{3}$ Animal Behavior Graduate Group, University of California, Davis, Davis, CA. USA \\ ${ }^{4}$ Estación Científica COIBA-AIP, Ciudad del Saber, Clayton, Panamá, Republic of \\ Panama \\ ${ }^{5}$ Museum of Natural Science and Department of Biological Sciences, Louisiana State \\ University, Baton Rouge, LA, USA \\ E-mail: castillopedroluis94@gmail.com; cmmonteza@ucdavis.edu;
} ojohns7@1su.edu; gangehr@gmail.com.

orcid.org/0000-0002-4974-6247; orcid.org/0000-0003-2537-2065; orcid.org/00000002-1764-5708

\begin{abstract}
The Isthmus of Panama, the narrowest land bridge between North and South America, is surrounded by a large number of islands $(>1500)$ relatively close to the mainland. However, despite the potential role Panamanian islands have for the conservation of bird species, most avian surveys are conducted on the mainland. The islands of Jicarón and Jicarita, located in Coiba National Park, are of particular interest because these are the southernmost islands in the Republic of Panama. Additionally, previous avian surveys were restricted to Coiba Island, the largest of the park, leaving the avifauna in the rest of the islands poorly known. Here, we provide the first annotated checklist of birds for the islands of Jicarón and Jicarita, where we documented 115 and 53 species, respectively. Of these 23 species represent new records for the park. Further, we documented 10 endemic subspecies and 29 migratory species, of which 7 are warblers. The most frequently observed species were Brown-backed Dove (Leptotila plumbeiceps battyi), Rufous-tailed Hummingbird (Amazilia tzacatl) and Bananaquit
\end{abstract}


(Coereba flaveola). We highlight some species of interest and discuss the potential role of these islands for conservation.

\title{
KEY WORDS
}

Birds, conservation, warblers, islands, biodiversity.

\section{PRIMER LISTADO DE AVES EN JICARON Y JICARITA: LAS ISLAS MAS AL SUR DE LA REPÚBLICA DE PANAMÁ}

\begin{abstract}
RESUMEN
El Istmo de Panamá, el territorio más estrecho entre la conexión de Norte y Sur América, posee un alto número de islas $(>1500)$ en sus costas. Sin embargo, pese al potencial que estas islas representan para la conservación, la mayoría de los estudios de avifauna ocurren en tierra firme. Las islas de Jicarón y Jicarita, ubicadas en el Parque Nacional Coiba, son interesantes porque son las islas más al sur del pacífico panameño. Además, estudios previos de aves en este parque eran restringidos a la isla más grande, Isla Coiba, dejando así desconocida la avifauna del resto de las islas. Este estudio representa el primer listado de aves para Jicarón y Jicarita, donde documentamos 115 y 53 especies, respectivamente. De las cuales 23 especies representan nuevos registros para el parque. Además, registramos 10 subespecies endémicas y 29 migratorias, de las cuales 7 son reinitas. Las especies observadas con más frecuencia son la paloma de Coiba (Leptotila plumbeiceps battyi), amazilia de cola rufa (Amazilia tzacatl) y mielero común (Coereba flaveola). Resaltamos especies de interés y el potencial rol de estas islas para la conservación.
\end{abstract}

\section{PALABRAS CLAVES}

Aves, conservación, Parulidae, islas, biodiversidad.

\section{INTRODUCTION}

In contrast to the mainland, the geographic isolation of islands promotes a suite of ecological conditions that may result in speciation, thus endemism (Kier et al., 2009; Fordham and Brook, 2010), and/or behavioral and morphological adaptations. Moreover, islands warrant attention for bird conservation because they are home for about one fifth of the worldwide avifauna, of which nearly $40 \%$ are threatened (Johnson 
and Stattersfield, 1990; Biber, 2002; Trevino et al., 2007). The large number $(>1500)$ of islands surrounding the Panamanian Isthmus, on both the Atlantic and Pacific coasts, are interesting because they were isolated from the mainland only recently (12,000-20,000 years) (Olson, 1997). However, most avian surveys in Panama have been restricted to the mainland.

In Panama, the islands of Coiba National Park (CNP) are particularly important because they contain some of the last remaining lowland humid forests in the southwest Pacific of Panama (Milton and Mittermeier, 1977). This park is a 270,125-ha archipelago composed by 9 larger islands and more than 100 islets. The largest islands of the park, Coiba and Jicarón, are separated by $6-7 \mathrm{~km}$ and have been isolated from each other presumably for as long as the archipelago has been from the mainland. The floristic composition of both islands is nearly $50 \%$ different from the mainland (Ibáñez, 2011), and is distributed among various habitat types, that are critical for the resident birds in the park, as well as for migratory bird species that use these islands as stopover points and, potentially, wintering areas.

Previous bird surveys in CNP have been conducted mainly on Coiba (Wetmore 1957, 1965, 1968, 1972; Wetmore et al., 1984; Ibáñez and Cabot 1997), leaving the bird communities of the rest of the islands unstudied. Understanding the bird community assemblages in the islands of the Coiban archipelago is critical, for example, to identify evolutionary significant units (ESUs) of interest and enforce conservation efforts (Crandall et al., 2000). Here, we provide the first annotated checklist of birds for Jicarón and Jicarita, using the results of 8 expeditions between 2004 and 2019. We compare the composition of the avifauna between the two largest islands, Coiba and Jicarón, and describe some species of interest for conservation.

\section{METHODS}

\section{Study site}

CNP is characterized by a humid tropical monsoonic forest, a rainfall of up to $3,403 \mathrm{~mm} \mathrm{yr}^{-1}$, an average temperature of $26.4^{\circ} \mathrm{C}$, and a marked 
seasonality, where the dry season extends from mid-December to midApril (Cardiel et al. 1997; Glynn \& Wellington, 1983). The islands in this park were used as a prison colony between 1919 and 2004, and was declared a protected area in 1991. In 2005, the park was declared by UNESCO as a World Heritage Site and it is currently managed by MiAmbiente (the Panamanian Ministry of Environment) and the Coiba Committee, with representatives of different sectors.

We conducted bird surveys in two islands of the Coiban archipelago (Fig. 1): Jicarón (1,868 ha), the second largest island in the park, and Jicarita (136 ha), which is the southernmost point of the Republic of Panama (Ibáñez, 2011). Respectively, their highest elevation is 392 and $122 \mathrm{~m}$ above sea level. Both islands are surrounded by rocky coast and have a very steep terrain, limiting access to a few landing sites. Further, Jicarita island has a continuous rocky cliff on its southernmost part. The habitats surveyed included coast, forest edge and interior, up to $1 \mathrm{~km}$ into the forest, including coastal vegetation, dry, secondary, and mature forest (Ibáñez, 2011).

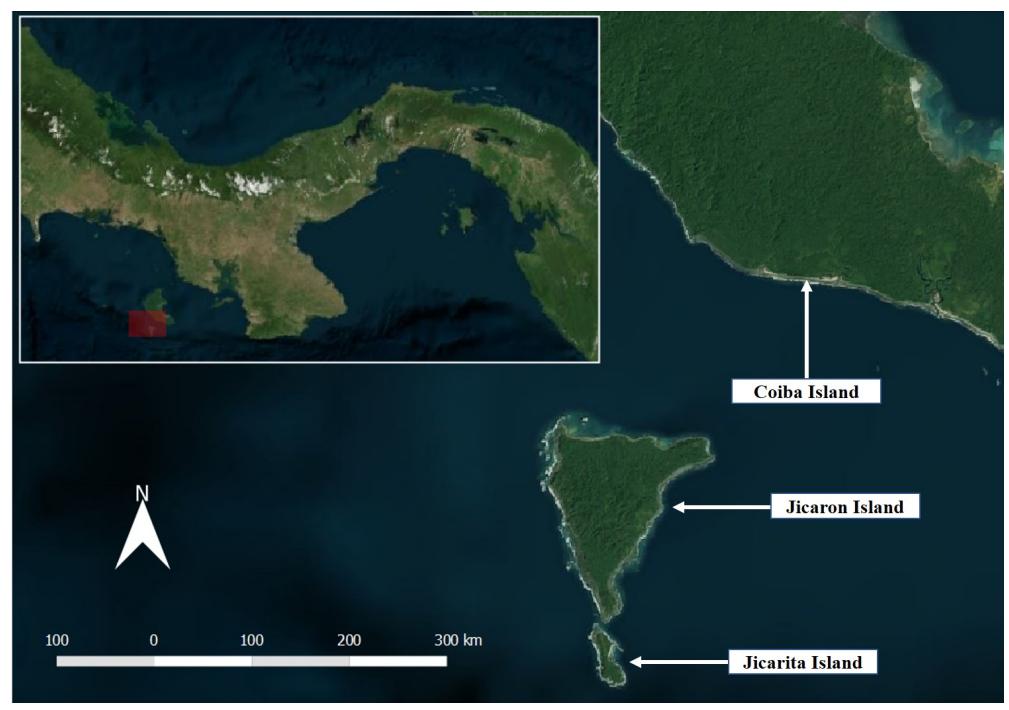

Fig. 1 Map showing the islands of Jicarón and Jicarita in Coiba National Park, in relation to the Republic of Panama (top left). Map was created using aGIS4 in QGIS 2.18.14 


\section{Data collection}

O. J. conducted a first bird surveys between 31 December, 2003 and 5 January, 2004 on the islands of Jicarón and Jicarita. G.R.A. obtained bird observations on both islands between 15 and 17 February, 2015. P.L.C.-C. and C.M.M-M. conducted surveys as part of a different project, with trips dates as follow: 23-31 July, 2017; 19-22 December, 2017; 3-7 January, 2018; 24-30 March, 2018; 12-13 April, 2019; and July 26-28, 2019. We made eight fieldtrips to Jicarón and three to Jicarita. Expeditions were conducted under research permit No. SE/APH-1-15 and SC/A-23-17 and corresponding renewals, approved by MiAmbiente.

We conducted bird observations in Jicarón via strip transects along the coast of the island, observation points (i.e., $7^{\circ} 17^{\prime} 26.4^{\prime \prime} \mathrm{N}, 81^{\circ} 48^{\prime} 15.6^{\prime \prime} \mathrm{W}$; $7^{\circ} 16^{\prime} 59.6^{\prime \prime} \mathrm{N}, 81^{\circ} 46^{\prime} 16.6^{\prime \prime} \mathrm{W}$; and $\left.7^{\circ} 13^{\prime} 41.8^{\prime \prime} \mathrm{N}, 81^{\circ} 48^{\prime} 10.7^{\prime \prime} \mathrm{W}\right)$ and opportunistic sightings. Some birds were observed, opportunistically, while going around the islands by boat and some were also photographed using camera traps (Reconyx PC900 Hyperfire Reconyx, Inc, WI, USA) used for a different project. On Jicarita, birds were observed on "observation points" (i.e., $7^{\circ} 13^{\prime} 13.6^{\prime \prime} \mathrm{N}$, $\left.81^{\circ} 48^{\prime} 08.1^{\prime \prime} \mathrm{W}\right)$ and an aquatic transect along the southernmost part of the island. Bird identifications were based on Angehr and Dean (2010), and the AOS Checklist (Chesser et al. 2019) for scientific and English names. Furthermore, to depict whether we have observed the complete species richness on Jicaron, we did a species richness accumulation curve using $\mathrm{R}$ package Vegan (Oksanen et al. 2019).

\section{RESULTS}

Overall, we recorded 115 species of birds on Jicarón (115) and Jicarita (53), from 39 and 22 families, respectively. The Jicarita list is a subset of that of Jicaron, with no species recorded from the former that was not also recorded from the latter. Our results include 23 new records for the CNP checklist. The most numerous family was Tyrannidae with 17 species, followed by Parulidae and Scolopacidae with 9 and 8 species, respectively. Further, we added 10 migratory bird species to the CNP checklist, making a total of 29 migratory bird species. The number of 
species shared between Coiba and Jicarón was 87.

Based on the Red List (IUCN 2019), two species on Jicaron and Jicarita are considered Near Threatened and one Vulnerable (Table 1). Further, our records expand the distributional range of the Bronzed Cowbird (Molothrus aeneus), Southern Lapwing (Vanellus chilensis), and Blackcrowned

Night-Heron

(Nycticorax nycticorax).

Table 1. List of the avifauna community at two islands of Coiba National Park (Jicarón and Jicarita). IUCN Status: LC - Least Concern (left as blank spaces); NT - Near Threatened; and VU - Vulnerable. The number of species per family are provided in brackets

\begin{tabular}{|c|c|c|c|c|}
\hline $\begin{array}{l}\text { Family/Scientific } \\
\text { name }\end{array}$ & & $\begin{array}{l}\text { Birdlife } \\
\text { Status }\end{array}$ & Jicarón & Jicarita \\
\hline \multicolumn{5}{|l|}{ Columbidae (2) } \\
\hline $\begin{array}{l}\text { Patagioenas } \\
\text { cayennensis } \\
\text { (Bonnaterre, 1792) }\end{array}$ & $\begin{array}{l}\text { Pale-vented } \\
\text { Pigeon }\end{array}$ & & $\mathrm{X}^{2,3,4,5,6,7,8,9}$ & \\
\hline $\begin{array}{l}\text { Leptotila } \\
\text { plumbeiceps } \\
\text { battyi* (Rothschild, } \\
1901)\end{array}$ & $\begin{array}{l}\text { Brown- } \\
\text { backed Dove }\end{array}$ & VU & $\mathrm{X}^{1,2,3,4,5,6,7,8,9}$ & $\mathrm{X}$ \\
\hline \multicolumn{5}{|l|}{ Cuculidae (1) } \\
\hline $\begin{array}{l}\text { Coccyzus minor } \\
\text { (Gmelin, 1788) }\end{array}$ & $\begin{array}{l}\text { Mangrove } \\
\text { Cuckoo }^{\dagger}\end{array}$ & & $\mathrm{X}^{1,3, \mathrm{~m}}$ & \\
\hline \multicolumn{5}{|l|}{ Caprimulgidae (1) } \\
\hline $\begin{array}{l}\text { Lurocalis } \\
\text { semitorquatus } \\
\text { (Gmelin, 1788) }\end{array}$ & $\begin{array}{l}\text { Short-tailed } \\
\text { Nighthawk }\end{array}$ & & $\mathrm{X}^{0}$ & \\
\hline $\begin{array}{l}\text { Antrostomus rufus } \\
\text { (Boddaert, 1783) }\end{array}$ & $\begin{array}{l}\text { Rufous } \\
\text { Nightjar }\end{array}$ & & $X^{3,4,9}$ & \\
\hline \multicolumn{5}{|l|}{ Apodidae (2) } \\
\hline $\begin{array}{l}\text { Chaetura pelagica } \\
\text { (Linnaeus, 1758) }\end{array}$ & $\begin{array}{l}\text { Chimney } \\
\text { Swift }^{\dagger}\end{array}$ & & $\mathrm{X}^{7, \mathrm{~m}}$ & \\
\hline $\begin{array}{l}\text { Chaetura vauxi } \\
\text { (Townsend, 1839) }\end{array}$ & Vaux's Swift & & $\mathrm{X}^{1,2,3,4,5,9}$ & $\mathrm{X}$ \\
\hline
\end{tabular}


Continue Table 1.

\begin{tabular}{|c|c|c|c|c|}
\hline $\begin{array}{l}\text { Family/Scientific } \\
\text { name }\end{array}$ & & $\begin{array}{l}\text { Birdlife } \\
\text { Status }\end{array}$ & Jicarón & Jicarita \\
\hline \multicolumn{5}{|l|}{ Trochilidae (4) } \\
\hline $\begin{array}{l}\text { Chlorostilbon } \\
\text { assimilis } \\
\text { (Lawrence, 1861) }\end{array}$ & $\begin{array}{l}\text { Garden } \\
\text { Emerald }\end{array}$ & & $\mathrm{X}^{1,2,3,4,6,7,8,9}$ & $X$ \\
\hline $\begin{array}{l}\text { Amazilia edward } \\
\text { (DeLattre \& } \\
\text { Bourcier, 1846) }\end{array}$ & $\begin{array}{l}\text { Snowy- } \\
\text { bellied } \\
\text { Hummingbird }\end{array}$ & & $X^{2,5,6}$ & \\
\hline $\begin{array}{l}\text { Amazilia tzacatl } \\
\text { (de la Llave, 1833) }\end{array}$ & $\begin{array}{l}\text { Rufous-tailed } \\
\text { Hummingbird }\end{array}$ & & $\mathrm{X}^{1,2,3,4,5,6,7,8,9}$ & $\mathrm{X}$ \\
\hline $\begin{array}{l}\text { Hylocharis eliciae } \\
\text { (Bourcier \& } \\
\text { Mulsant, 1846) }\end{array}$ & $\begin{array}{l}\text { Blue-throated } \\
\text { Goldentail }\end{array}$ & & $\mathrm{X}^{0,1,2,3,4,6,8,9}$ & $X$ \\
\hline \multicolumn{5}{|l|}{ Rallidae (1) } \\
\hline $\begin{array}{l}\text { Aramides cajaneus } \\
\text { (Müller, 1776) }\end{array}$ & $\begin{array}{l}\text { Gray-cowled } \\
\text { Wood-Rail }\end{array}$ & & $X^{2,9}$ & \\
\hline \multicolumn{5}{|l|}{$\begin{array}{l}\text { Haematopodidae } \\
\text { (1) }\end{array}$} \\
\hline $\begin{array}{l}\text { Haematopus } \\
\text { palliatus } \\
\text { (Temminck, 1820) }\end{array}$ & $\begin{array}{l}\text { American } \\
\text { Oystercatcher }\end{array}$ & & $\mathrm{X}^{0,1,2,3,4,5,6,7,8,9}$ & $X$ \\
\hline \multicolumn{5}{|l|}{ Charadriidae (4) } \\
\hline $\begin{array}{l}\text { Vanellus chilensis } \\
\text { (Molina, 1782) }\end{array}$ & $\begin{array}{l}\text { Southern } \\
\text { Lapwing }^{\dagger}\end{array}$ & & $X^{4}$ & \\
\hline $\begin{array}{l}\text { Charadrius } \\
\text { collaris (Vieillot, } \\
1818 \text { ) }\end{array}$ & $\begin{array}{l}\text { Collared } \\
\text { Plover }^{\dagger}\end{array}$ & & $X^{4}$ & \\
\hline $\begin{array}{l}\text { Charadrius } \\
\text { wilsonia (Ord, } \\
1814)\end{array}$ & $\begin{array}{l}\text { Wilson's } \\
\text { Plover }\end{array}$ & & $X^{4 m}$ & \\
\hline $\begin{array}{l}\text { Charadrius } \\
\text { semipalmatus } \\
\text { (Bonaparte, 1825) }\end{array}$ & $\begin{array}{l}\text { Semipalmated } \\
\text { Plover }\end{array}$ & & $X^{1,3 m}$ & \\
\hline
\end{tabular}


Cotinue Table 1.

\begin{tabular}{|c|c|c|c|c|}
\hline $\begin{array}{l}\text { Family/Scientific } \\
\text { name }\end{array}$ & & $\begin{array}{l}\text { Birdlife } \\
\text { Status }\end{array}$ & Jicarón & Jicarita \\
\hline \multicolumn{5}{|l|}{ Scolopacidae (9) } \\
\hline $\begin{array}{l}\text { Numenius phaeopus } \\
\text { (Linnaeus, 1758) }\end{array}$ & Whimbrel & & $X^{0,2,4,5,7 m}$ & $\mathrm{X}$ \\
\hline $\begin{array}{l}\text { Arenaria interpres } \\
\text { (Linnaeus, 1758) }\end{array}$ & $\begin{array}{l}\text { Ruddy } \\
\text { Turnstone }\end{array}$ & & $X^{4,7 m}$ & \\
\hline $\begin{array}{l}\text { Calidris minutilla } \\
\text { (Vieillot, 1819) }\end{array}$ & $\begin{array}{l}\text { Least } \\
\text { Sandpiper }\end{array}$ & & $X^{4,7 m}$ & \\
\hline $\begin{array}{l}\text { Calidris pusilla } \\
\text { (Linnaeus, 1766) }\end{array}$ & $\begin{array}{l}\text { Semipalmated } \\
\text { Sandpiper }\end{array}$ & NT & $X^{3,7 m}$ & \\
\hline $\begin{array}{l}\text { Calidris mauri } \\
\text { (Cabanis, 1857) }\end{array}$ & $\begin{array}{l}\text { Western } \\
\text { Sandpiper }\end{array}$ & & $X^{3,7 m}$ & \\
\hline $\begin{array}{l}\text { Tringa incana } \\
\text { (Gmelin, 1789) }\end{array}$ & $\begin{array}{l}\text { Wandering } \\
\text { Tattler }\end{array}$ & & $X^{7 m}$ & \\
\hline $\begin{array}{l}\text { Phalaropus lobatus } \\
\text { (Linnaeus, 1758) }\end{array}$ & $\begin{array}{l}\text { Red-necked } \\
\text { Phalarope }^{\dagger}\end{array}$ & & $X^{3 m}$ & \\
\hline $\begin{array}{l}\text { Actitis macularius } \\
\text { (Linnaeus, 1766) }\end{array}$ & $\begin{array}{l}\text { Spotted } \\
\text { Sandpiper }\end{array}$ & & $\mathrm{X}^{0,1,2,3,4,5,6,8,9}$ & $\mathrm{X}$ \\
\hline $\begin{array}{l}\text { Tringa solitaria } \\
\text { (Wilson, 1813) }\end{array}$ & $\begin{array}{l}\text { Solitary } \\
\text { Sandpiper }\end{array}$ & & $X^{7 m}$ & \\
\hline \multicolumn{5}{|l|}{ Laridae (5) } \\
\hline $\begin{array}{l}\text { Leucophaeus } \\
\text { atricilla (Linnaeus, } \\
1758 \text { ) }\end{array}$ & $\begin{array}{l}\text { Laughing } \\
\text { Gull }\end{array}$ & & $X^{6,8,9}$ & \\
\hline $\begin{array}{l}\text { Anous stolidus } \\
\text { (Linnaeus, 1758) }\end{array}$ & Brown Noddy & & $X^{2,5,7}$ & \\
\hline $\begin{array}{l}\text { Onychoprion } \\
\text { anaethetus } \\
\text { (Scopoli, 1786) }\end{array}$ & Bridled Tern & & $X^{8}$ & \\
\hline $\begin{array}{l}\text { Chlidonias niger } \\
\text { (Linnaeus, 1758) }\end{array}$ & Black Tern & & $X^{3,5 m}$ & \\
\hline $\begin{array}{l}\text { Thalasseus } \\
\text { maximus (Boddaert, } \\
\text { 1783) }\end{array}$ & Royal Tern & & $\mathrm{X}^{1,2,3,4,6,7,9}$ & $X$ \\
\hline
\end{tabular}


Continue Table 1.

\begin{tabular}{|c|c|c|c|c|}
\hline $\begin{array}{l}\text { Family/Scientific } \\
\text { name }\end{array}$ & & $\begin{array}{l}\text { Birdlife } \\
\text { Status }\end{array}$ & Jicarón & Jicarita \\
\hline \multicolumn{5}{|l|}{$\begin{array}{l}\text { Procellariidae (1) } \\
\text { (1) }\end{array}$} \\
\hline $\begin{array}{l}\text { Puffinus subalaris } \\
\text { (Ridgway, 1897) }\end{array}$ & $\begin{array}{l}\text { Galapagos } \\
\text { Shearwater }^{\dagger}\end{array}$ & & $X^{2,5 m}$ & $\mathrm{X}$ \\
\hline \multicolumn{5}{|l|}{ Hydrobatidae (1) } \\
\hline $\begin{array}{l}\text { Oceanodroma tethys } \\
\text { (Bonaparte, 1852) }\end{array}$ & $\begin{array}{l}\text { Wedge-rumped } \\
\text { Storm-Petrel }\end{array}$ & & $\mathrm{X}^{2,8 \mathrm{~m}}$ & \\
\hline \multicolumn{5}{|c|}{ Fregatidae (1) } \\
\hline $\begin{array}{l}\text { Fregata magnificens } \\
\text { (Mathews, 1914) }\end{array}$ & $\begin{array}{l}\text { Magnificent } \\
\text { Frigatebird }\end{array}$ & & $\mathrm{X}^{0,1,2,3,4,5,6.8,9}$ & $\mathrm{X}$ \\
\hline \multicolumn{5}{|c|}{ Sulidae (2) } \\
\hline $\begin{array}{l}\text { Sula leucogaster } \\
\text { (Boddaert, 1783) }\end{array}$ & Brown Booby & & $\mathrm{X}^{1,2,3,4,5,6,7,8,9}$ & $\mathrm{X}$ \\
\hline \multicolumn{5}{|l|}{$\begin{array}{l}\text { Phalacrocoracidae } \\
\text { (1) }\end{array}$} \\
\hline $\begin{array}{l}\text { Phalacrocorax } \\
\text { brasilianus (Gmelin, } \\
\text { 1789) }\end{array}$ & $\begin{array}{l}\text { Neotropic } \\
\text { Cormorant }\end{array}$ & & $X^{2,3,5,9}$ & $\mathrm{X}$ \\
\hline \multicolumn{5}{|l|}{ Pelecanidae (1) } \\
\hline $\begin{array}{l}\text { Pelecanus } \\
\text { occidentalis } \\
\text { (Linnaeus, 1766) }\end{array}$ & Brown Pelican & & $\mathrm{X}^{0,1,2,3,4,5,6,7,8,9}$ & $\mathrm{X}$ \\
\hline \multicolumn{5}{|l|}{ Ardeidae (7) } \\
\hline $\begin{array}{l}\text { Tigrisoma } \\
\text { mexicanum } \\
\text { (Swainson, 1834) }\end{array}$ & $\begin{array}{l}\text { Bare-throated } \\
\text { Tiger-Heron }\end{array}$ & & $\mathrm{X}^{0,1,2,3,4,5,6,7,8,9}$ & $\mathrm{X}$ \\
\hline $\begin{array}{l}\text { Ardea herodias } \\
\text { (Linnaeus, 1758) }\end{array}$ & $\begin{array}{l}\text { Great Blue } \\
\text { Heron }\end{array}$ & & $X^{1,3}$ & \\
\hline $\begin{array}{l}\text { Ardea alba } \\
\text { (Linnaeus, 1758) }\end{array}$ & Great Egret & & $X^{3,4}$ & $\mathrm{X}$ \\
\hline $\begin{array}{l}\text { Egretta thula } \\
\text { (Molina, 1782) }\end{array}$ & Snowy Egret & & $\mathrm{X}^{0}$ & \\
\hline $\begin{array}{l}\text { Bubulcus ibis } \\
\text { (Linnaeus, 1758) }\end{array}$ & Cattle Egret ${ }^{\dagger}$ & & $X^{1,2,4,5}$ & \\
\hline $\begin{array}{l}\text { Nyctanassa violacea } \\
\text { (Linnaeus, 1758) }\end{array}$ & $\begin{array}{l}\text { Yellow- } \\
\text { crowned } \\
\text { Night-Heron }\end{array}$ & & $X^{2,9}$ & \\
\hline $\begin{array}{l}\text { Nycticorax nycticorax } \\
\text { (Linnaeus, 1758) }\end{array}$ & $\begin{array}{l}\text { Black-crowned } \\
\text { Night-Heron }{ }^{\dagger}\end{array}$ & & $\mathrm{X}^{3}$ & \\
\hline
\end{tabular}


Continue Table 1.

\begin{tabular}{|c|c|c|c|c|}
\hline $\begin{array}{l}\text { Family/Scientific } \\
\text { name }\end{array}$ & & $\begin{array}{l}\text { Birdlife } \\
\text { Status }\end{array}$ & Jicarón & Jicarita \\
\hline \multicolumn{5}{|l|}{$\begin{array}{l}\text { Threskiornithidae } \\
\text { (1) }\end{array}$} \\
\hline $\begin{array}{l}\text { Eudocimus albus } \\
\text { (Linnaeus, 1758) }\end{array}$ & White Ibis & & $\mathrm{X}^{0,2,5,7}$ & $\mathrm{X}$ \\
\hline \multicolumn{5}{|l|}{ Cathartidae (3) } \\
\hline $\begin{array}{l}\text { Coragyps atratus } \\
\text { (Bechstein, 1783) }\end{array}$ & $\begin{array}{l}\text { Black } \\
\text { Vulture }\end{array}$ & & $\mathrm{X}^{1,2,3,4,5,6,7,8,9}$ & $\mathrm{X}$ \\
\hline $\begin{array}{l}\text { Cathartes aura } \\
\text { (Linnaeus, 1758) }\end{array}$ & $\begin{array}{l}\text { Turkey } \\
\text { Vulture }\end{array}$ & & $\mathrm{X}^{0,1,2,3,4,5,6,8,9}$ & $\mathrm{X}$ \\
\hline $\begin{array}{l}\text { Sarcoramphus papa } \\
\text { (Linnaeus, 1758) }\end{array}$ & $\begin{array}{l}\text { King } \\
\text { Vulture }\end{array}$ & & $\mathrm{X}^{0,1,3,4,6,7,8,9}$ & $\mathrm{X}$ \\
\hline \multicolumn{5}{|l|}{ Pandionidae (1) } \\
\hline $\begin{array}{l}\text { Pandion haliaetus } \\
\text { (Linnaeus, 1758) }\end{array}$ & Osprey & & $\mathrm{X}^{0,1,2,3,4,6,7,9}$ & $\mathrm{X}$ \\
\hline \multicolumn{5}{|l|}{ Accipitridae (8) } \\
\hline $\begin{array}{l}\text { Harpagus bidentatus } \\
\text { (Latham, 1790) }\end{array}$ & $\begin{array}{l}\text { Double- } \\
\text { toothed Kite }\end{array}$ & & $\mathrm{X}^{3,9}$ & $X$ \\
\hline $\begin{array}{l}\text { Buteogallus } \\
\text { anthracinus (Deppe, } \\
\text { 1830) }\end{array}$ & $\begin{array}{l}\text { Common } \\
\text { Black Hawk }\end{array}$ & & $\mathrm{X}^{0,1,2,3,4,5,6,7,8,9}$ & $\mathrm{X}$ \\
\hline $\begin{array}{l}\text { Buteogallus } \\
\text { urubitinga (Gmelin, } \\
\text { 1788) }\end{array}$ & $\begin{array}{l}\text { Great Black } \\
\text { Hawk }^{\dagger}\end{array}$ & & $X^{5,8}$ & \\
\hline $\begin{array}{l}\text { Rupornis magnirostris } \\
\text { (Gmelin, 1788) }\end{array}$ & $\begin{array}{l}\text { Roadside } \\
\text { Hawk }\end{array}$ & & $\mathrm{X}^{1,9}$ & \\
\hline $\begin{array}{l}\text { Pseudastur albicollis } \\
\text { (Latham, 1790) }\end{array}$ & White Hawk & & $\mathrm{X}^{2}$ & \\
\hline $\begin{array}{l}\text { Buteo brachyurus } \\
\text { (Vieillot, 1816) }\end{array}$ & $\begin{array}{l}\text { Short-tailed } \\
\text { Hawk }^{\dagger}\end{array}$ & & $X^{3,5,8}$ & $\mathrm{X}$ \\
\hline $\begin{array}{l}\text { Buteo jamaicensis } \\
\text { (Gmelin, 1788) }\end{array}$ & $\begin{array}{l}\text { Red-tailed } \\
\text { Hawk }^{\dagger}\end{array}$ & & $\mathrm{X}^{0,3 \mathrm{~m}}$ & $X$ \\
\hline $\begin{array}{l}\text { Buteo albonotatus } \\
\text { (Kaup, 1847) }\end{array}$ & $\begin{array}{l}\text { Zone-tailed } \\
\text { Hawk }^{\dagger}\end{array}$ & & $\mathrm{X}^{3}$ & \\
\hline
\end{tabular}


Continue Table 1.

\begin{tabular}{|c|c|c|c|c|}
\hline $\begin{array}{l}\text { Family/Scientific } \\
\text { name }\end{array}$ & & $\begin{array}{c}\text { Birdlife } \\
\text { Status } \\
\end{array}$ & Jicarón & Jicarita \\
\hline \multicolumn{5}{|l|}{ Strigidae (1) } \\
\hline $\begin{array}{l}\text { Megascops choliba } \\
\text { (Vieillot, 1817) }\end{array}$ & $\begin{array}{l}\text { Tropical } \\
\text { Screech- } \\
\text { Owl }\end{array}$ & & $X^{1,4,5,8,9}$ & $X$ \\
\hline \multicolumn{5}{|l|}{ Alcedinidae (2) } \\
\hline $\begin{array}{l}\text { Megaceryle torquata } \\
\text { (Linnaeus, 1766) }\end{array}$ & $\begin{array}{l}\text { Ringed } \\
\text { Kingfisher }\end{array}$ & & $\mathrm{X}^{4,5}$ & \\
\hline $\begin{array}{l}\text { Chloroceryle } \\
\text { americana (Gmelin, } \\
1788)\end{array}$ & $\begin{array}{l}\text { Green } \\
\text { Kingfisher }\end{array}$ & & $\mathrm{X}^{1,2,3,4,5,6,8,9}$ & $X$ \\
\hline \multicolumn{5}{|l|}{ Picidae (1) } \\
\hline $\begin{array}{l}\text { Melanerpes } \\
\text { rubricapillus } \\
\text { (Cabanis, 1862) }\end{array}$ & $\begin{array}{l}\text { Red- } \\
\text { crowned } \\
\text { Woodpecker }\end{array}$ & & $\mathrm{X}^{0,1,2,3,4,5,6,7,8,9}$ & $X$ \\
\hline \multicolumn{5}{|l|}{ Falconidae (3) } \\
\hline $\begin{array}{l}\text { Milvago chimachima } \\
\text { (Vieillot, 1816) }\end{array}$ & $\begin{array}{l}\text { Yellow- } \\
\text { headed } \\
\text { Caracara }\end{array}$ & & $X^{2,3,4,9}$ & $X$ \\
\hline $\begin{array}{l}\text { Falco columbarius } \\
\text { (Linnaeus, 1758) }\end{array}$ & $\operatorname{Merlin}^{\dagger}$ & & $\mathrm{X}^{0, \mathrm{~m}}$ & $\mathrm{X}$ \\
\hline $\begin{array}{l}\text { Falco peregrinus } \\
\text { (Tunstall, 1771) }\end{array}$ & $\begin{array}{l}\text { Peregrine } \\
\text { Falcon }\end{array}$ & & $\mathrm{X}^{3,4,6,9 \mathrm{~m}}$ & $\mathrm{X}$ \\
\hline \multicolumn{5}{|l|}{ Psittacidae (5) } \\
\hline $\begin{array}{l}\text { Ara macao } \\
\text { (Linnaeus, 1758) }\end{array}$ & $\begin{array}{l}\text { Scarlet } \\
\text { Macaw }\end{array}$ & & $X^{4}$ & \\
\hline $\begin{array}{l}\text { Brotogeris jugularis } \\
\text { (Muller, 1776) }\end{array}$ & $\begin{array}{l}\text { Orange- } \\
\text { chinned } \\
\text { Parakeet }\end{array}$ & & $\mathrm{X}^{1,2,3,4,5,7,8,9}$ & $\mathrm{X}$ \\
\hline $\begin{array}{l}\text { Pionus menstruus } \\
\text { (Linnaeus, 1766) }\end{array}$ & $\begin{array}{l}\text { Blue-headed } \\
\text { Parrot }\end{array}$ & & $X^{2,4,5,9}$ & \\
\hline $\begin{array}{l}\text { Amazona autumnalis } \\
\text { (Linnaeus, 1758) }\end{array}$ & $\begin{array}{l}\text { Red-lored } \\
\text { Parrot }\end{array}$ & & $\mathrm{X}^{1,2,3,4,5,6,7,8,9}$ & $X$ \\
\hline $\begin{array}{l}\text { Amazona farinosa } \\
\text { (Boddaert, 1783) }\end{array}$ & $\begin{array}{l}\text { Mealy } \\
\text { Parrot }\end{array}$ & NT & $X^{4,9}$ & \\
\hline
\end{tabular}


Continue Table 1.

\begin{tabular}{|c|c|c|c|c|}
\hline $\begin{array}{l}\text { Family/Scientific } \\
\text { name }\end{array}$ & & $\begin{array}{l}\text { Birdlife } \\
\text { Status }\end{array}$ & Jicarón & Jicarita \\
\hline \multicolumn{5}{|l|}{ Thamnophilidae (1) } \\
\hline $\begin{array}{l}\text { Thamnophilus } \\
\text { doliatus* (Linnnaeus, } \\
\text { 1764) }\end{array}$ & $\begin{array}{l}\text { Barred } \\
\text { Antshrike }\end{array}$ & & $\mathrm{X}^{1,2,3,4,5,6,7,8,9}$ & $\mathrm{X}$ \\
\hline \multicolumn{5}{|l|}{ Tyrannidae (17) } \\
\hline $\begin{array}{l}\text { Capsiempis flaveola } \\
\text { (Lichtenstein, 1823) }\end{array}$ & $\begin{array}{l}\text { Yellow } \\
\text { Tyrannulet }\end{array}$ & & $X^{3}$ & $X$ \\
\hline $\begin{array}{l}\text { Myiopagis viridicata } \\
\text { (Vieillot, 1817) }\end{array}$ & $\begin{array}{l}\text { Greenish } \\
\text { Elaenia }\end{array}$ & & $\mathrm{X}^{0,1,2,3,5,8,9}$ & $\mathrm{X}$ \\
\hline $\begin{array}{l}\text { Elaenia flavogaster } \\
\text { (Thunberg, 1822) }\end{array}$ & $\begin{array}{l}\text { Yellow- } \\
\text { bellied } \\
\text { Elaenia }\end{array}$ & & $\mathrm{X}^{1,6,7,9}$ & $\mathrm{X}$ \\
\hline $\begin{array}{l}\text { Elaenia chiriquensis } \\
\text { (Lawrence, 1865) }\end{array}$ & $\begin{array}{l}\text { Lesser } \\
\text { Elaenia }\end{array}$ & & $X^{1,5}$ & \\
\hline $\begin{array}{l}\text { Mionectes oleagineus } \\
\text { (Lichtenstein, 1823) }\end{array}$ & $\begin{array}{l}\text { Ochre- } \\
\text { bellied } \\
\text { Flycatcher }\end{array}$ & & $X^{2}$ & \\
\hline $\begin{array}{l}\text { Contopus sordidulus } \\
\text { (Sclater, 1859) }\end{array}$ & $\begin{array}{l}\text { Western } \\
\text { Wood- } \\
\text { Pewee }\end{array}$ & & $X^{4 m}$ & \\
\hline $\begin{array}{l}\text { Contopus cinereus* } \\
\text { (von Spix, 1825) }\end{array}$ & $\begin{array}{l}\text { Tropical } \\
\text { Pewee }\end{array}$ & & $X^{2,9}$ & \\
\hline $\begin{array}{l}\text { Empidonax sp. } \\
\text { (Cabanis, 1855) }\end{array}$ & $\begin{array}{l}\text { Flycatcher } \\
\text { sp. }\end{array}$ & & $X^{4}$ & \\
\hline $\begin{array}{l}\text { Myiarchus } \\
\text { tuberculifer } \\
\text { (D’Orbigny \& } \\
\text { Lafresnaye, 1837) }\end{array}$ & $\begin{array}{l}\text { Dusky- } \\
\text { capped } \\
\text { Flycatcher }\end{array}$ & & $X^{2,3,4,5}$ & \\
\hline $\begin{array}{l}\text { Myiarchus } \\
\text { panamensis } \\
\text { (Lawrence, 1860) }\end{array}$ & $\begin{array}{l}\text { Panama } \\
\text { Flycatcher }\end{array}$ & & $\mathrm{X}^{0,1,2,3,4,5,6,7,8,9}$ & $\mathrm{X}$ \\
\hline $\begin{array}{l}\text { Myiarchus crinitus } \\
\text { (Linnaeus, 1758) }\end{array}$ & $\begin{array}{l}\text { Great } \\
\text { Crested } \\
\text { Flycatcher }\end{array}$ & & $X^{3,7 m}$ & $\mathrm{X}$ \\
\hline
\end{tabular}


Continue Table 1.

\begin{tabular}{|c|c|c|c|c|}
\hline $\begin{array}{l}\text { Family/Scientific } \\
\text { name }\end{array}$ & & $\begin{array}{l}\text { Birdlife } \\
\text { Status }\end{array}$ & Jicarón & Jicarita \\
\hline $\begin{array}{l}\text { Myiodynastes } \\
\text { maculatus (Statius } \\
\text { Müller, 1776) }\end{array}$ & $\begin{array}{l}\text { Streaked } \\
\text { Flycatcher }\end{array}$ & & $X^{4,7,9}$ & $X$ \\
\hline $\begin{array}{l}\text { Myiodynastes } \\
\text { luteiventris (Sclater, } \\
1859 \text { ) }\end{array}$ & $\begin{array}{l}\text { Sulphur- } \\
\text { bellied } \\
\text { Flycatcher }^{\dagger}\end{array}$ & & $\mathrm{X}^{3, \mathrm{~m}}$ & \\
\hline $\begin{array}{l}\text { Tyrannus } \\
\text { melancholicus } \\
\text { (Vieillot, 1819) }\end{array}$ & $\begin{array}{l}\text { Tropical } \\
\text { Kingbird }\end{array}$ & & $\mathrm{X}^{1,2,3,4,5,6,7,8,9}$ & $X$ \\
\hline $\begin{array}{l}\text { Tyrannus verticalis } \\
\text { (Say, 1823) }\end{array}$ & $\begin{array}{l}\text { Western } \\
\text { Kingbird }^{\dagger}\end{array}$ & & $X^{3, m}$ & \\
\hline $\begin{array}{l}\text { Tityridae (1) } \\
\text { Tityra semifasciata } \\
\text { (Spix, 1825) }\end{array}$ & $\begin{array}{l}\text { Masked } \\
\text { Tityra }\end{array}$ & & $X^{1,4,7,9}$ & \\
\hline $\begin{array}{l}\text { Vireonidae (1) } \\
\text { Vireo flavoviridis } \\
\text { (Cassin, 1851) } \\
\text { Hirundinidae (4) }\end{array}$ & $\begin{array}{l}\text { Yellow- } \\
\text { green Vireo }\end{array}$ & & $\mathrm{X}^{1,3,4,7,9}$ & $X$ \\
\hline $\begin{array}{l}\text { Progne chalybea } \\
\text { (Gmelin, 1789) }\end{array}$ & $\begin{array}{l}\text { Gray- } \\
\text { breasted } \\
\text { Martin }\end{array}$ & & $\mathrm{X}^{1,3,4,5,7,8,9}$ & \\
\hline $\begin{array}{l}\text { Tachycineta } \\
\text { albilinea } \\
\text { (Lawrence, 1863) }\end{array}$ & $\begin{array}{l}\text { Mangrove } \\
\text { Swallow }\end{array}$ & & $X$ & $X$ \\
\hline $\begin{array}{l}\text { Hirundo rustica } \\
\text { (Linnaeus, 1758) }\end{array}$ & $\begin{array}{l}\text { Barn } \\
\text { Swallow }\end{array}$ & & $X^{4, m}$ & \\
\hline $\begin{array}{l}\text { Stelgidopteryx } \\
\text { ruficollis (Vieillot, } \\
1817 \text { ) }\end{array}$ & $\begin{array}{l}\text { Southern } \\
\text { Rough- } \\
\text { winged } \\
\text { Swallow }\end{array}$ & & $X^{3,4,9}$ & \\
\hline $\begin{array}{l}\text { Troglodytidae (1) } \\
\text { Troglodytes aedon* } \\
\text { (Vieillot, 1809) } \\
\text { Polioptilidae (1) }\end{array}$ & $\begin{array}{l}\text { House } \\
\text { Wren }\end{array}$ & & $\mathrm{X}^{0,1,2,3,4,5,6,7,8,9}$ & $X$ \\
\hline $\begin{array}{l}\text { Polioptila } \\
\text { plumbea* (Gmelin, } \\
1788)\end{array}$ & $\begin{array}{l}\text { Tropical } \\
\text { Gnatcatcher }\end{array}$ & & $\mathrm{X}^{1,2,3,4,5,6,7,8,9}$ & $X$ \\
\hline
\end{tabular}

Tecnociencia, Vol. 22, $N^{\circ} 2$ 
Continue Table 1.

\begin{tabular}{|c|c|c|c|c|}
\hline $\begin{array}{l}\text { Family/Scientific } \\
\text { name }\end{array}$ & & $\begin{array}{l}\text { Birdlife } \\
\text { Status }\end{array}$ & Jicarón & Jicarita \\
\hline \multicolumn{5}{|l|}{ Turdidae (2) } \\
\hline $\begin{array}{l}\text { Catharus ustulatus } \\
\text { (Nuttall, 1840) }\end{array}$ & $\begin{array}{l}\text { Swainson's } \\
\text { Thrush }\end{array}$ & & $\mathrm{X}^{7 \mathrm{~m}}$ & \\
\hline $\begin{array}{l}\text { Turdus assimilis* } \\
\text { (Cabanis, 1850) }\end{array}$ & $\begin{array}{l}\text { White- } \\
\text { throated } \\
\text { Thrush }\end{array}$ & & $\mathrm{X}^{1,2,3,4,5,7,8,9}$ & \\
\hline \multicolumn{5}{|l|}{ Passerellidae (1) } \\
\hline $\begin{array}{l}\text { Arremonops } \\
\text { conirostris* } \\
\text { (Bonaparte, 1850) }\end{array}$ & $\begin{array}{l}\text { Black-striped } \\
\text { Sparrow }\end{array}$ & & $\mathrm{X}^{1,2,4,5,7,9}$ & \\
\hline \multicolumn{5}{|l|}{ Icteridae (2) } \\
\hline $\begin{array}{l}\text { Quiscalus mexicanus } \\
\text { (Gmelin, 1788) }\end{array}$ & $\begin{array}{l}\text { Great-tailed } \\
\text { Grackle }\end{array}$ & & $X^{2,3,4,5,7,9}$ & \\
\hline $\begin{array}{l}\text { Molothrus aeneus } \\
\text { (Wagler, 1829) }\end{array}$ & $\begin{array}{l}\text { Bronzed } \\
\text { Cowbird }^{\dagger}\end{array}$ & & $\mathrm{X}^{2}$ & \\
\hline \multicolumn{5}{|l|}{ Parulidae (9) } \\
\hline $\begin{array}{l}\text { Parkesia motacilla } \\
\text { (Vieillot, 1809) }\end{array}$ & $\begin{array}{l}\text { Louisiana } \\
\text { Waterthrush }^{\dagger}\end{array}$ & & $X^{3,4,7 m}$ & \\
\hline $\begin{array}{l}\text { Parkesia } \\
\text { noveboracensis } \\
\text { (Gmelin, 1789) }\end{array}$ & $\begin{array}{l}\text { Northern } \\
\text { Waterthrush }\end{array}$ & & $\mathrm{X}^{0,3,6,7,9 \mathrm{~m}}$ & $X$ \\
\hline $\begin{array}{l}\text { Leiothlypis peregrina } \\
\text { (Wilson, 1811) }\end{array}$ & $\begin{array}{l}\text { Tennessee } \\
\text { Warbler }\end{array}$ & & $\mathrm{X}^{1, \mathrm{~m}}$ & \\
\hline $\begin{array}{l}\text { Geothlypis } \\
\text { philadelphia (Wilson, } \\
1810 \text { ) }\end{array}$ & $\begin{array}{l}\text { Mourning } \\
\text { Warbler }^{\dagger}\end{array}$ & & $X^{4 m}$ & \\
\hline $\begin{array}{l}\text { Geothylpis formosa } \\
\text { (Wilson, 1811) }\end{array}$ & $\begin{array}{l}\text { Kentucky } \\
\text { Warbler }\end{array}$ & & $\mathrm{X}^{7}$ & $X$ \\
\hline
\end{tabular}


Continue Table 1.

\begin{tabular}{|c|c|c|c|c|}
\hline $\begin{array}{l}\text { Family/Scientific } \\
\text { name }\end{array}$ & & $\begin{array}{l}\text { Birdlife } \\
\text { Status }\end{array}$ & Jicarón & Jicarita \\
\hline $\begin{array}{l}\text { Setophaga pitiayumi* } \\
\text { (Vieillot, 1817) }\end{array}$ & $\begin{array}{l}\text { Tropical } \\
\text { Parula }\end{array}$ & & $\mathrm{X}^{0,1,2,3,5,7,9}$ & $\mathrm{X}$ \\
\hline $\begin{array}{l}\text { Setophaga petechia } \\
\text { (Linnaeus, 1766) }\end{array}$ & $\begin{array}{l}\text { Yellow } \\
\text { Warbler }\end{array}$ & & $\mathrm{X}^{1,2,3,4,5,6,7,8,9}$ & $\mathrm{X}$ \\
\hline $\begin{array}{l}\text { Basileuterus rufifrons } \\
\text { (Swainson, 1838) }\end{array}$ & $\begin{array}{l}\text { Rufous- } \\
\text { capped } \\
\text { Warbler }\end{array}$ & & $X^{2,3,7}$ & $\mathrm{X}$ \\
\hline $\begin{array}{l}\text { Cardellina } \\
\text { canadensis (Linnaeus, } \\
1766 \text { ) }\end{array}$ & $\begin{array}{l}\text { Canada } \\
\text { Warbler }^{\dagger}\end{array}$ & & $X^{7 m}$ & \\
\hline \multicolumn{5}{|l|}{ Cardinalidae (1) } \\
\hline $\begin{array}{l}\text { Piranga rubra } \\
\text { (Linnaeus, 1758) }\end{array}$ & $\begin{array}{l}\text { Summer } \\
\text { Tanager }\end{array}$ & & $X^{7 m}$ & \\
\hline \multicolumn{5}{|l|}{ Thraupidae (5) } \\
\hline $\begin{array}{l}\text { Coereba flaveola } \\
\text { (Linnaeus, 1758) }\end{array}$ & Bananaquit & & $\mathrm{X}^{0,1,2,3,4,5,6,7,8,9}$ & $\mathrm{X}$ \\
\hline $\begin{array}{l}\text { Ramphocelus } \\
\text { dimidiatus* } \\
\text { (Lafresnaye, 1837) }\end{array}$ & $\begin{array}{l}\text { Crimson- } \\
\text { backed } \\
\text { Tanager }\end{array}$ & & $\mathrm{X}^{0,1,2,3,4,5,6,7,8,9}$ & $\mathrm{X}$ \\
\hline $\begin{array}{l}\text { Thraupis episcopus* } \\
\text { (Linnaeus, 1766) }\end{array}$ & $\begin{array}{l}\text { Blue-gray } \\
\text { Tanager }\end{array}$ & & $\mathrm{X}^{0,1,2,3,4,5,6,7,8,9}$ & $\mathrm{X}$ \\
\hline $\begin{array}{l}\text { Thraupis palmarum } \\
\text { (Wied, 1821) }\end{array}$ & Palm Tanager & & $X^{4,8}$ & \\
\hline $\begin{array}{l}\text { Cyanerpes cyaneus } \\
\text { (Linnaeus, 1766) }\end{array}$ & $\begin{array}{l}\text { Red-legged } \\
\text { Honeycreeper }\end{array}$ & & $X^{1,3,4,9}$ & \\
\hline
\end{tabular}

Legend description:

\footnotetext{
$*=$ subspecies $\quad 5^{5}=$ July, 2018 expedition

${ }^{\dagger}=$ new records for Coiba National Park $\quad{ }^{6}=$ December, 2018 expedition

${ }^{0}=$ December/January, 2003/2004 expedition $\quad{ }^{7}=$ March, 2019 expedition

${ }^{1}=$ Coiba Bioblitz expedition, $2015 \quad{ }^{8}=$ July, 2019 expedition

${ }^{2}=$ July, 2017 expedition $\quad 9^{9}=$ February, 2020 expedition

${ }^{3}=$ December, $2017-$ January 2018 expedition $\quad \mathrm{m}=$ Migratory

${ }^{4}=$ March, 2018 expedition $\quad b=$ Migratory breeding
} 
Notes on some species of special interest.

Black-crowned Night-Heron (Nycticorax nycticorax Linnaeus, 1758): during the trip in December, 2017, we observed a single adult individual flying near our camping area. This is the first record of this species in CNP and reports of this species are uncommon in the region (see Angehr and Dean, 2010).

Bronzed cowbird (Molothrus aeneus Wagler, 1829): this species is considered to be common on the eastward Pacific slopes of Panama. Individual were observed in Jicarón Island, representing a distribution expansion of this species.

Grey-headed (Brown-backed) Dove (Leptotila plumbeiceps battyi): this form (together with the subspecies malae of the Azuero Peninsula) is considered a full species by most references (e.g. Wetmore 1968; Monroe and Sibley 1993; Baptista et al., 1997; Gibbs et al., 2001; Dickinson 2003; Birdlife International, 2019) other than AOS (Chesser et al., 2019). It was common on Jicaron and also encountered on Jicarita. It is otherwise restricted to Coiba, Cébaco, and the Azuero Peninsula (Wetmore, 1968) and Rancheria (G.R.A. pers. obsn). It is considered Vulnerable by BirdLife International (2019).

Mealy Parrot (Amazona farinosa Boddaert, 1783): this species is considered Near Threatened. On Jicarón Island, we observed a small group ( $<12$ individuals) of Mealy Parrots foraging in the forest canopy and vocalizing. Sightings occurred on the expedition of July, 2017 in mature forest at about $1 \mathrm{~km}$ from the coast.

Scarlet macaw (Ara macao Linnaeus, 1758): we observed individuals of Scarlet Macaw in the island of Jicarón, during the visit in March, 2018. Individuals were observed in Punta Ursula, the southern tip of Jicarón Island $\left(7^{\circ} 13^{\prime} 41.8^{\prime \prime} \mathrm{N}, 81^{\circ} 48^{\prime} 10.7^{\prime \prime} \mathrm{W}\right)$, foraging seeds of the tropical almond (Terminalia catappa). Considering this species was not observed in any other field trip, we presumed these individuals fly from Coiba Island when this valuable resource is available (Fig. 2). 


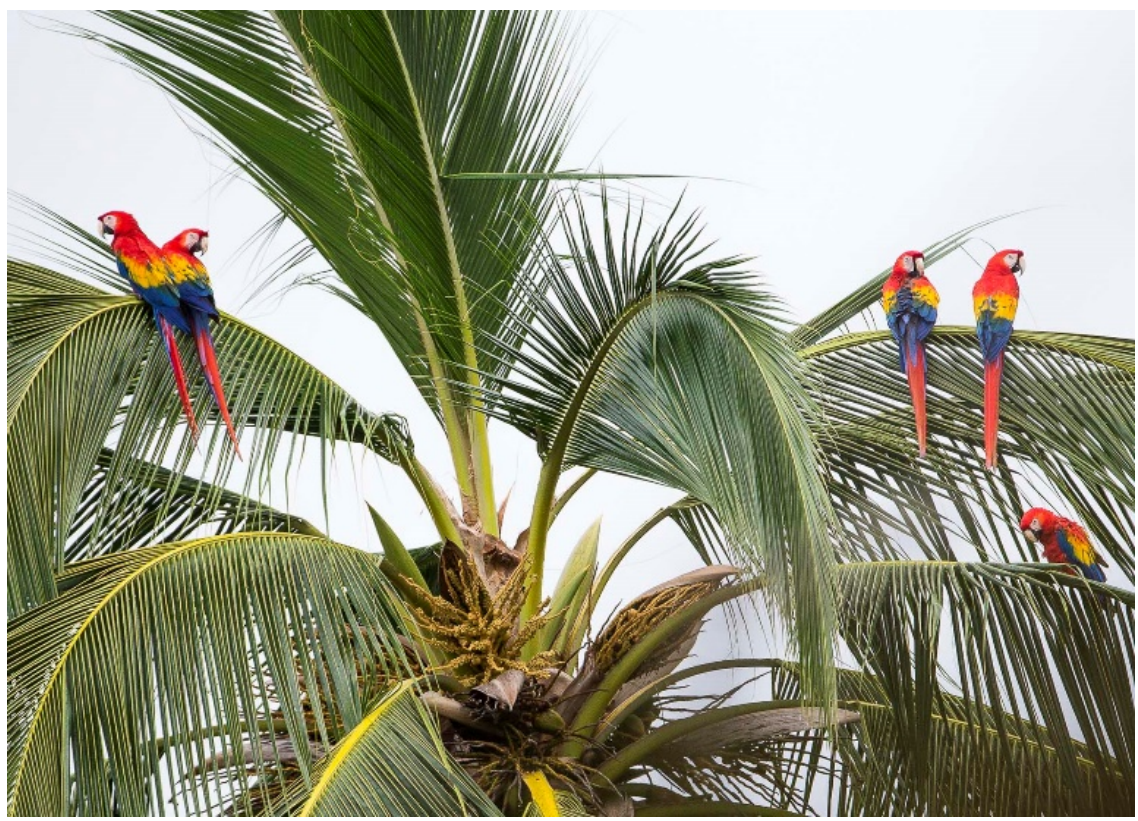

Fig. 2 Scarlet Macaw (Ara macao). Photo credit: Christian Ziegler

Wandering Tattler (Tringa incana Gmelin, 1789): this small shorebird is a rare transient, with few sightings in Panama, most records being from the Frijoles islets off the north coast of Coiba (i.e. eBird: checklist S27159064 by Groenendijk, 2016). We observed a single individual along the rocky shore in the northwest section of Jicaron.

Western Kingbird (Tyrannus verticalis Say, 1823): we recorded a single individual of this vagrant species on December 20, 2017. The individual was on coastal vegetation (Fig. 3) near Punta Ursula. In general, this species is rarely documented in Panama and our observation represents the southernmost record for this species, replacing its previous report of the locality El Chorogo, in the Province of Chiriqui (eBird: checklist S21016561 by Montanez, 1999). 


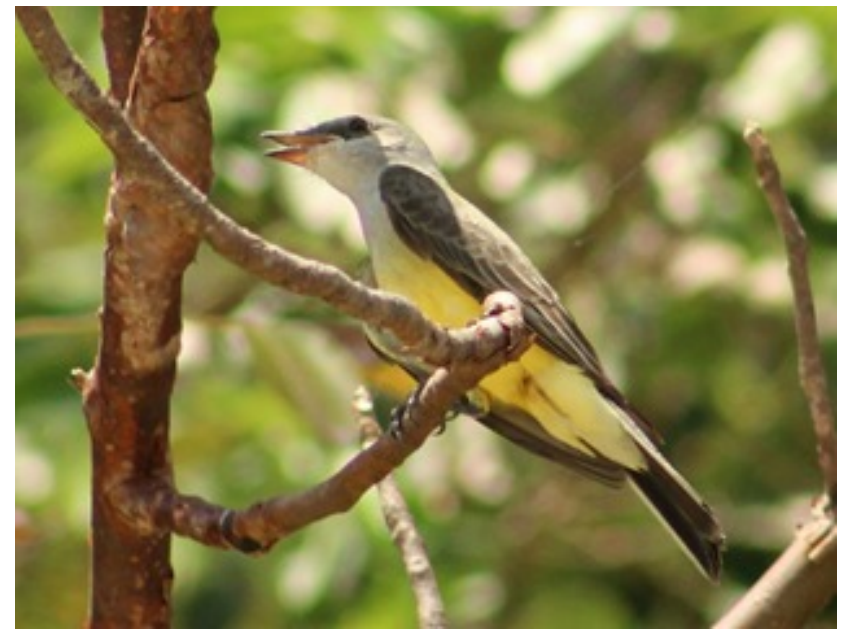

Fig. 3 Western Kingbird (Tyrannus verticalis). Photo credit: PLC

Yellow-crowned Night-Heron (Nyctanassa violacea Linnaeus, 1758): the only record of this species was taken by a camera trap in Punta Ursula of Jicarón Island. The photographed individual was an immature (Fig. 4) and represents the southernmost record for this species in Panama. This species was first recorded by first Wetmore (1957) in Coiba Island and recent reports are uncommon.

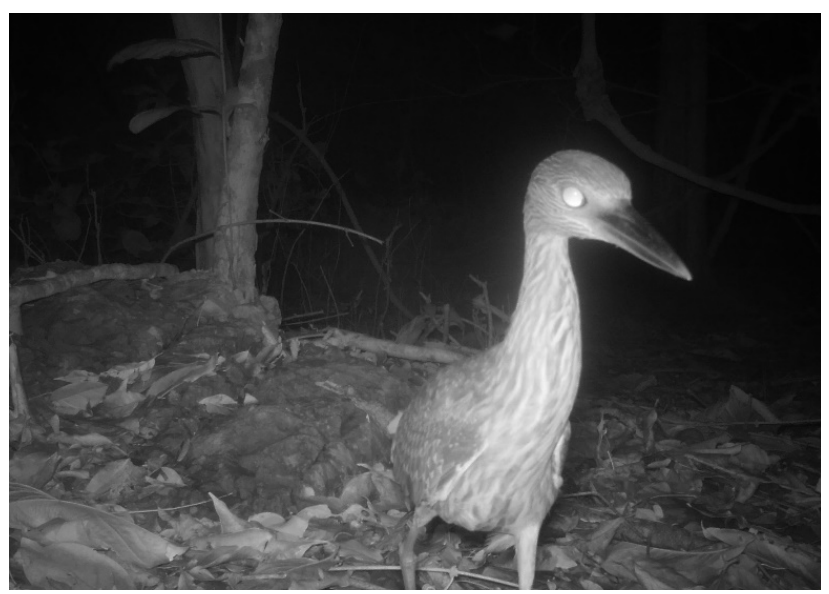

Fig. 4 Yellow-crowned Night-Heron (Nyctanassa violacea). Photo credit: CMMM 
Yellow-headed Caracara (Milvago chimachima Vieillot, 1816): this species was first recorded on Coiba in 1993 and since 2001 has become common throughout the island (G.R.A. pers. obsn.). In the past 30 years, the species has undergone a remarkable range extension throughout Panama (Fig. 5)

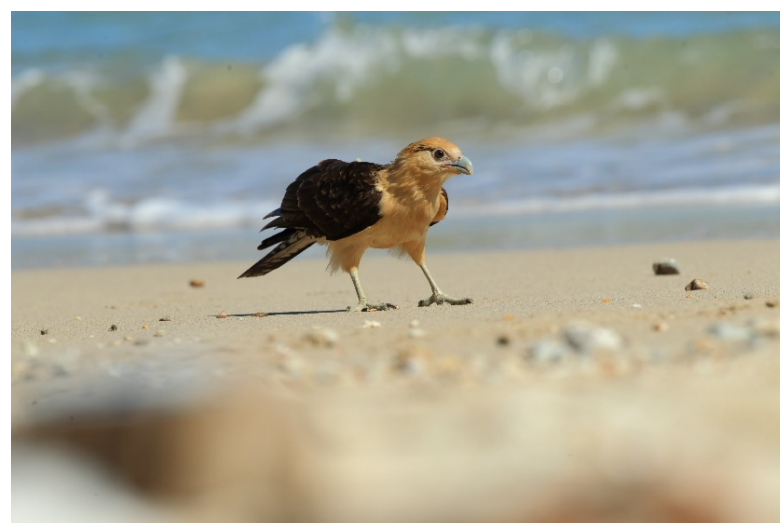

Fig. 5 Yellow-headed Caracara (Milvago chimachima). Photo credit: Christian Ziegler

\section{DISCUSSION}

This study represents the first annotated checklist of birds for Jicarón and Jicarita. We documented 115 species of birds from 39 families in Jicarón and 53 species from 22 families in Jicarita. We recorded 23 bird species that have not been observed on Coiba or other islands of the archipelago, representing new records for CNP (Ibáñez and Cabot 1997). Hence, our results enlarge the reported avifauna of CNP to 191 species, from 45 families. Our findings indicate that $10.4 \%$ of the Panamanian avifauna has been recorded on Jicarón and Jicarita, including 11 of the 20 endemic subspecies of Coiba, plus 29 migratory species (67 for CNP), 4 transient, 2 vagrant and 1 breeding migratory (Angehr and Dean, 2010).

The largest numbers of species recorded corresponded to the family Tyrannidae with 17 species, followed by Parulidae and Scolopacidae with eight species each. Among our observations, the most commonly 
observed species on Jicarón were Grey-headed (Brown-backed Dove) (Leptotila plumbeiceps battyi), Rufous-tailed Hummingbird (Amazilia tzacatl), and Bananaquit (Coereba flaveola).

We did not record Coiba Spinetail (Cranioleuca dissita) on either island. This species is thus presumably restricted to Coiba and Ranchería alone. Likewise, we did not record the Coiba endemic subspecies of Scalybreasted Hummingbird (Phaeochroa curvierii saturatior), Southern Beardless-Tyrannulet (Camptostoma obsoletum orphnum), Sepiacapped Flycatcher (Leptopogon amaurocephalus idius), Scrub Greenlet (Hylophilus flavipes xuthus), Rufous-browed Peppershrike (Cyclarhis gujanensis coibae), Tropical Parula (Parula pitiayumi cirrha), Streaked Saltator (Saltator albicollis scotinus), Yellow-faced Grassquit (Tiaris olivacea raviola), or Thick-billed Seed-Finch (Oryzoborus angolensis fractor).

We only recorded four nocturnal species, including Rufous Nightjar (Antrostomus rufus), Yellow-Crowned Night-Heron (Nyctanassa violacea), Black-Crowned Night-Heron (Nycticorax nycticorax), and the Tropical Screech-Owl (Megascops choliba). However, we only recorded nocturnal species from at or near our camping site, or that were photographed by camera traps, which function continuously throughout the day and night. Although our camera trapping effort is significant ( $\sim 5000$ trap-nights), camera traps were mounted on trees at $50 \mathrm{~cm}$ above the ground and would not record most arboreal species. Thus, there are potentially more nocturnal bird species Jicarón Island.

Including our checklist, there are currently 67 migratory birds known for the park, of which 16 species are warblers (Angehr and Dean, 2010), indicating that $37 \%$ of the migratory warblers recorded for Panama use Coiba National Park during their migration, and 20\% use Jicarón. These records suggest that the Coiban archipelago may play a role as a stopover point for migratory bird species. In contrast with the mainland in Panama (Fischer and Vasseur, 2002), the islands of Coiba National Park provide stopover points with reduced anthropogenic effects, since there are no human settlements other than the Ministry of Environment visitors center and the Police station on Coiba Island and the 
Smithsonian field station on Ranchería Island.

With regard to seabirds, we noted a colony of $>200$ individuals of Brown Boobies nesting in rocky cliffs at the southernmost point of the island (Fig. 6). This represents the largest colony of Brown Boobies in the archipelago. Angehr et al. (2004) reported a colony of 120 Brown Boobies and 80 Brown Pelicans nesting at this site, as well as a nesting colony of 28 Brown Boobies on Uva Island in the Contreras Islands in the northern part of CNP.

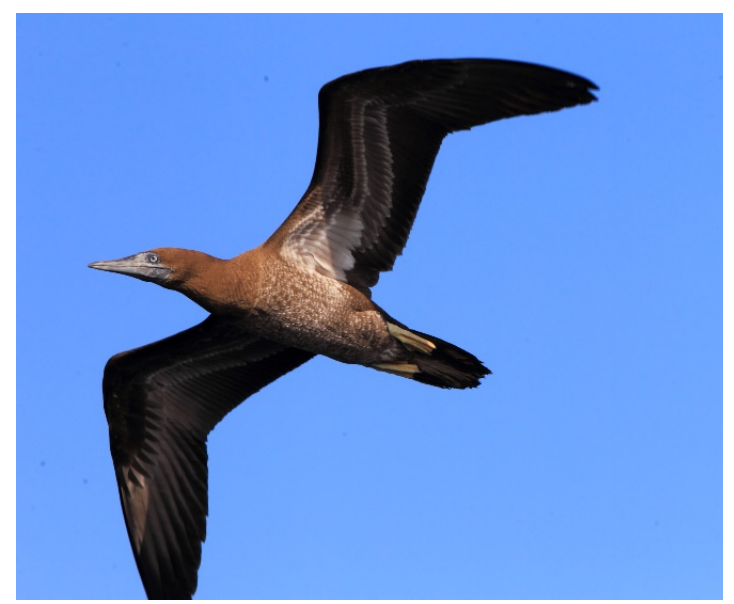

Fig. 6 Brown Boobies (Sula leucogaster). Photo credit: Christian Ziegler

While our list includes 53 species of birds for Jicarita, this may not represent the complete checklist as we made only short visits to the island in three occasions. Our checklist is the result of eight short expeditions; however, there are a number of sites on these islands, particularly Jicarón, that we have not visited due to logistic and access constraints. Therefore, the avifauna on both islands may be larger than our results suggest, particularly for Jicarón. For instance, using $\mathrm{R}$ package Vegan (Oksanen et al. 2019), we produced a species richness accumulation curve only for Jicarón (Fig. 7), but the curve does not reach an asymptote, indicating that our surveys did not fully sample the avifauna of these islands. 


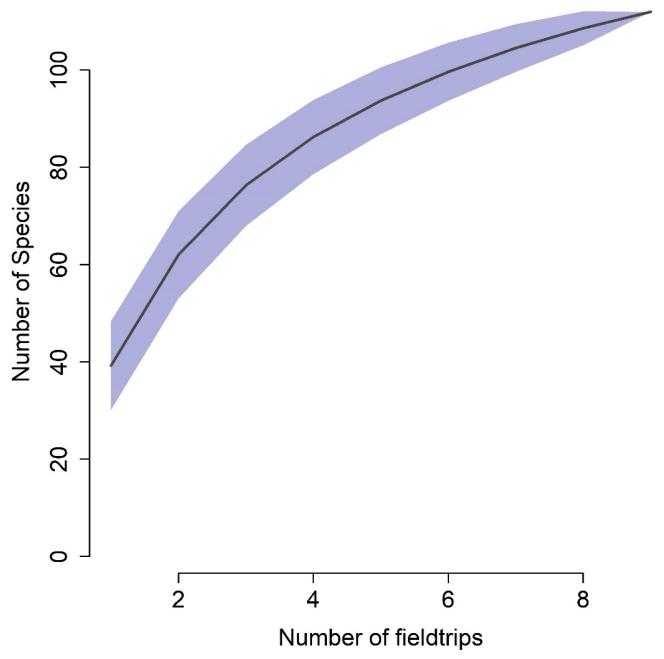

Fig. 7 Species richness accumulation curve for Jicarón Island, based on field observations

Our data indicate that species richness similarity between Jicarón and Coiba is about $45 \%$, although the latter is 27 times larger. Thus, the islands of CNP, along other mainland sites (i.e. Bahia Honda and Cerro Hoya), offer an opportunity for studying population dynamics, migration patterns, and behavioral adaptations (Wright and Steadman, 2012) between the islands of CNP and the mainland. The annotated checklist we present here for Jicarón and Jicarita contribute to the baseline information of bird community composition in CNP. We hope our records contribute to an improvement of management policies and, conservation awareness for CNP, and encourage more bird community monitoring on the islands of CNP, as well as other important protected areas in the mainland of the Pacific of Panama such as the sky-island of Cerro Hoya and of Bahia Honda (Buitrago-Rosas et al. 2019; Jimenez and Aparicio, 2005).

\section{ACKNOWLEDGMENTS}

We thank Omar Lopez, Valerie McMillan, and especially Christian Ziegler for logistic support during the 2015 Coiba Bioblitz, as well as 
all sponsors of this expedition. Logistic support was also provided by Smithsonian Tropical Research Institute. We would like to acknowledge Meg Crofoot and Brendan Barrett for enabling us to participate in expeditions in 2017 and 2019 that were part of a different project, which was made possible thanks to a Coss Award for International Field Research awarded to Brendan Barrett and CMMM and a L.S.B. Leakey Foundation grant awarded to Brendan Barrett. Support was also provided to CMMM by a Packard Foundation Fellowship (2016-65130) awarded to Meg Crofoot. CMMM is funded by SENACYT, scholarship program number BIDP-2017-2018. We are grateful to Brendan Barrett, Zarluis M. Mijango, and Evelyn del Rosario for company in the field, and especially to our boat driver Eliecer Vega for transporting us wherever it is needed in CNP. OJ is supported by the National Science Foundation Graduate Research Fellowship under grant no. DGE1247192.

\section{REFERENCES}

Angehr, G. R., \& Dean, R. (2010). The birds of Panama: a field guide. Cornell University Press, Ithaca NY.

Angehr, G. R., \& Dean, R. (2010). The birds of Panama: a field guide. A Zona Tropical Publication from Comstock Publishing Associates, Cornell University Press.

Biber, E., 2002. Patterns of endemic extinctions among island bird species. Ecography. 25(6), pp.661-676.

BirdLife International (2018) IUCN Red List for birds. Downloaded from http://www.birdlife.org on 28/07/2018.

Buitrago-Rosas, D., Medina, J. L., Castillo-Caballero, P. L., Ortega, J., Garzón, J. L. \& Falk, J. J., 2019. Highland avian surveys in Cerro Hoya National Park (Azuero, Panamá) reveal new range extensions, including a rare hummingbird (Selasphorus sp.). Ornitología Neotropical, 30, pp.89-97.

Cardiel, J. M., Castroviejo, S. \& Velayos, M., 1997. El Parque Nacional 
Coiba: El medio físico. In: Castroviejo, S. (Ed.), Flora y Fauna del Parque Nacional de Coiba (Panama). Serviprint Press, Madrid, pp. 1130.

Crandall, K. A., Bininda-Emonds, O. R., Mace, G. M. \& Wayne, R. K., 2000. Considering evolutionary processes in conservation biology. Trends in ecology \& evolution, 15(7), pp.290-295.

Chesser, R. T., K. J. Burns, C. Cicero, J. L. Dunn, A. W. Kratter, I. J Lovette, P. C. Rasmussen, J.V. Remsen, J. D. Rising, D. F. Stotz \& K. Winker (2016) Fiftyseventh Supplement to the American Ornithologists' Union: Checklist of North American Birds. The Auk 133: 544-560.

Fischer, A. \& Vasseur, L., 2002. Smallholder perceptions of agroforestry projects in Panama. Agroforestry systems, 54(2), pp.103-113. Fordham, D. A. \& Brook, B. W., 2010. Why tropical islands endemics are acutely susceptible to global change. Biodiversity and conservation, 19(2), pp. 329-342.

Glynn, P. W., Wellington, G. M., Glynn, O. \& Wells, J. W., 1983. Corals and coral reefs of the Galapagos Islands. University of California Press, California.

Groenendijk, K., 2016. eBird checklist: http://ebird.org/ebird/view/checklist?subID=S27159064. eBird, Ithaca, New York, USA. Available at http://www.ebird.org [Accessed 22 March 2020].

Ibáñez, A., 2011. Guía botánica del Parque nacional Coiba. International Cooperative Biodiversity Groups: Instituto Smithsonian de Investigaciones Tropicals: Secretaría Nacional de C.

Ibáñez, C., \& Cabot, J., (1997). Las aves del Parque Nacional de Coiba (Panamá). In:Castroviejo, Santiago (Eds.). Flora y Fauna del Parque Nacional de Coiba (Panamá). pp. 457-467.

Jimenez B., \& Aparicio (2005), K. Birds of Bahia Honda (Veraguas, Panama). In:Castroviejo, S., \& Ibáñez, A. (Eds.). Estudios sobre la 
biodiversidad de la región de Bahía Honda (Veraguas, Panamá) (Vol. 20). Editorial CSIC-CSIC Press.

Johnson, T. H. \& Stattersfield, A. J., 1990. A global review of island endemic birds. Ibis, 132(2), pp.167-180.

Kier, G., Kreft, H., Lee, T. M., Jetz, W., Ibisch, P. L., Nowicki, C., Mutke, J. \& Barthlott, W., 2009. A global assessment of endemism and species richness across island and mainland regions. Proceedings of the National Academy of Sciences, 106(23), pp.9322-9327.

Méndez-Carvajal, P. G. (2012). Population study of Coiba howler monkeys (Alouatta coibensis coibensis) and Coiba capuchin monkeys (Cebus capucinus imitator), Coiba Island National Park, Republic of Panama. Journal of primatology., l(2), 104.

Miller, M. J., Angehr, G. R., Ridgely, R. S., Klicka, J., Ch, O. G. L., Arauz, J., Campos, C. E. \& Buitrago-Rosas, D. (2015). Annotated checklist of the birds (Aves) of Cerro Hoya National Park, Azuero Peninsula, Panamá. Check List, 11(2), 1585.

Milton, K., \& Mittermeier, R. A. (1977). A brief survey of the primates of Coiba Island, Panama. Primates, 18(4), 931-936.

Montanez, D., $1999 . \quad$ eBird checklist: http://ebird.org/ebird/view/checklist?subID=S21016561. eBird, Ithaca, New York, USA. Available at http://www.ebird.org [Accessed 22 March 2020].

Oksanen, J., Blanchet, F. G., Friendly, M., Kindt, R., Legendre, P., McGlinn, D., Minchin, P. R., O'Hara, R. B., Simpson, G. L., Solymos, P. \& Stevens, M. H. H., 2018. vegan: Community Ecology Package. R package version 2.5-2. 2018.

Olson, S. L., 1997. Avian biogeography in the islands of the Pacific Coast of western Panama. The Era of Allan R. Phillips: A Festschrift.

Reyes, D., Alcalá, R. E., Arias, D., \& Osorio-Beristain, M. (2009). 
Genetic structuring at a fine scale in the Russet-crowned Motmot (Momotus mexicanus) in a tropical dry forest in central Mexico. Western North American Naturalist,69(2), 171-174.

Sanderson, J. G., \& Trolle, M. (2005). Monitoring elusive mammals: unattended cameras reveal secrets of some of the world's wildest places. American Scientist, 93(2), 148-155.

Thornton, D. H., Branch, L. C., \& Sunquist, M. E. (2012). Response of large galliforms and tinamous (Cracidae, Phasianidae, Tinamidae) to habitat loss and fragmentation in northern Guatemala. Oryx, 46(4), 567576.

Trevino, H. S., Skibiel, A. L., Karels, T. J. \& Dobson, F. S., 2007. Threats to avifauna on oceanic islands. Conservation Biology, 21(1), pp.125-132.

Weckel, M., Giuliano, W., \& Silver, S. (2006). Jaguar (Panthera onca) feeding ecology: distribution of predator and prey through time and space. Journal of Zoology, 270(1), 25-30.

Wetmore, A., 1957. The birds of isla Coiba, Panamá. Smithsonian Miscellaneous Collections.

Wetmore, A., 1965. The birds of the Republic of Panama. Part 1: Tinamidae (Tinamous) to Rynchopidae (Skimmers). Smithsonian Miscellaneous Collections, Volume 150. Smithsonian Institution Press, Washington, D. C., USA.

Wetmore, A., 1968. The birds of the Republic of Panama. Part 2: Columbidae (Pigeons) to Picidae (Woodpeckers). Smithsonian Miscellaneous Collections, Volume 150. Smithsonian Institution Press, Washington, D. C., USA.

Wetmore, A., 1972. The birds of the Republic of Panama. Part 3. Passeriformes: Dendrocolaptidae (Woodcreepers) to Oxyruncidae (Sharpbills). Smithsonian Miscellaneous Collections, Volume 150. 
Smithsonian Institution Press, Washington, D. C., USA.

Wetmore, A., Pasquier, R. F., \& Olson, S. L. 1984. The Birds of the Republic of Panama. Part 4. Hirundinadae (Swallows) to Fringillidae (Finches). Smithsonian Institution: Washington D. C.

Wright, N. A. \& Steadman, D. W., 2012. Insular avian adaptations on two Neotropical continental islands. Journal of biogeography, 39(10), pp.1891-1899.

Recibido 29 de febrero de 2020, aceptado 26 de marzo de 2020. 